\title{
Characterising the big pieces of Lipschitz graphs property using projections
}

\section{Martikainen, Henri}

2018

Martikainen , H \& Orponen , T 2018 , ' Characterising the big pieces of Lipschitz graphs property using projections ' , European Mathematical Society. Journal , vol. 20 , no. 5 , pp. 1055-1073 . https://doi.org/10.4171/JEMS/782

http://hdl.handle.net/10138/307144

https://doi.org/10.4171/JEMS/782

cc_by_nc

acceptedVersion

Downloaded from Helda, University of Helsinki institutional repository.

This is an electronic reprint of the original article.

This reprint may differ from the original in pagination and typographic detail.

Please cite the original version. 


\title{
CHARACTERISING THE BIG PIECES OF LIPSCHITZ GRAPHS PROPERTY USING PROJECTIONS
}

\author{
HENRI MARTIKAINEN AND TUOMASORPONEN
}

\begin{abstract}
A BSTRACT. We characterise the big pieces of Lipschitz graphs property in terms of projections. Roughly speaking, we prove that if a large subset of an n-A hlforsDavid regular set $E \quad R^{d}$ has plenty of projections in $L^{2}$, then a large part of $E$ is contained in a single Lipschitz graph. This is closely related to a question of G. David and S. Semmes.
\end{abstract}

\section{INTRODUCTION AND STATEMENT OF RESULTS}

The purpose of this paper is to characterise the big pieces of Lipschitz graphs (BPLG) condition in terms of projections, and only projections. We begin with some definitions, and then formulate the characterisation. After that, we will discuss the context of the result and provide an outline of the proof.

We are concerned with $n$-A hlfors-David regular sets in $\mathrm{R}^{\mathrm{d}}$ :

Definition 1.1 (n-ADR). Given $n 2 N$, a set $E \quad R^{d}$ is $n$-A hlfors-David regular (n-ADR) if $\mathrm{C}_{1} \mathrm{r}^{\mathrm{n}} \quad \mathrm{H}^{\mathrm{n}}(\mathrm{E} \backslash \mathrm{B}(\mathrm{x} ; \mathrm{r})) \quad \mathrm{C}_{2} \mathrm{r}^{\mathrm{n}}$ for all points $\times 2 \mathrm{E}$ and radii $\mathrm{r} 2$ $(0 ; \operatorname{diam}(E)]$, and some constants $0<C_{1} \quad C_{2}<1$. The constants $C_{1} ; C_{2}$ are referred to as the ADR constants of $E$.

With an $\mathrm{n}$-dimensional Lipschitz graph $={ }_{\mathrm{A}}$ in $\mathrm{R}^{\mathrm{d}}$ wemean a set of the form

$$
=f p+A(p): p 2 P g ;
$$

where $P \quad R^{d}$ is an $n$-dimensional subspace and $A$ : $P$ ! $P$ ? is a Lipschitz map. By the Lipschitz constant of , we mean the Lipschitz constant Lip(A) of A. Our main object of study is the following subclass of n-ADR sets:

Definition 1.2 (BPLG). An n-ADR set $E \quad R^{d}$ has big pieces of Lipschitz graphs, if there exist constants $M<1$ and $>0$ with the following property: for every $x 2 \mathrm{E}$ and $\mathrm{r} 2(0$; diam(E)], there exists an n-dimensional Lipschitz graph $\quad$ x;r with Lipschitz constant at most $M$, such that $H^{n}(E \backslash \quad x ; r \backslash B(x ; r)) \quad r^{n}$.

The closely related uniformly rectifiablesets are defined as follows:

2010 M athematics Subject Classification. 28 A 75.

Key words and phrases. Projections, Lipschitz graphs, Big pieces of sets, Uniform rectifiability.

H.M. is supported by the A cademy of Finland through the grant Multiparameter dyadic harmonic analysis and probabilistic methods.

T.O. is supported by the A cademy of Finland through the grant Restricted families of projections and connections to Kakeya type problems. 
Definition 1.3 (n-UR). A closed set $E \quad R^{d}$ is $n$-uniformly rectifiable (n-UR) if it is $\mathrm{n}-\mathrm{ADR}$, and there exist constants $>0$ and $M<1$ with the following property: for every $\times 2 \mathrm{E}$ and $\mathrm{r} 2(0$; $\operatorname{diam}(\mathrm{E})]$ there is a Lipschitz mapping $g: B_{R^{n}}(0 ; r) ! R^{d}$ such that $L i p(g) \quad M$ and

$$
H^{n}\left(E \backslash B(x ; r) \backslash g\left(B_{R^{n}}(0 ; r)\right)\right) \quad r^{n}:
$$

For the basics of UR and BPLG sets, we refer to the monographs [2,3] of David and Semmes. Notice that BPLG trivially implies UR. However, the converse is not true by an unpublished example of T. H rycak (see the discussion after Theorem 1.6). Let us also mention the recent deep geometric result by J. Azzam and R. Schul [1], which says that UR $=(B P)^{2} L G$, that is, UR sets contain big pieces of sets which have BPLG.

We need the following final definitions. For any $1 \quad k<d$, denote by $G(d ; k)$ the Grassmannian manifold of all $k$-dimensional subspaces of $R^{d}$, equipped with the distance $k V \quad W k_{G(d ; k)}:=k v \quad w k$. Here $v$ is the orthogonal projection onto $\mathrm{V}$ and $\mathrm{k} k$ is the usual operator norm of linear mappings. There is also a natural Borel probability measure d;k on $G(d ; k)$ - see Chapter 3 of [7]. Closed metric balls on $G(d ; k)$ are usually denoted by $B(V ; r)$, or $B_{G(d ; k)}(V ; r)$, if there is any risk of confusion.

The next proposition gives an easy necessary condition for a set to have BPLG:

Proposition 1.4. Assumethat $E \quad R^{d}$ has BPLG. Then, thereexist constants $>0$ and $C<1$ (depending only on $M$ and in thedefinition of BPLG) such that thefollowing holds: for every $x 2 \mathrm{E}$, and every radius $\mathrm{r} 2(0 ; \operatorname{diam}(\mathrm{E})]$, thereexists a subspace $\mathrm{V}_{\mathrm{x} ; \mathrm{r}} 2$ $G(d ; n)$ and a subset $E_{x ; r} \quad E \backslash B(x ; r)$ with theproperties that $H^{n}\left(E_{x ; r}\right) \quad r^{n}=C$, and

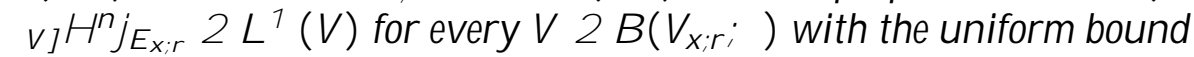

$$
k_{v]} H^{n^{n}} j_{E_{x ; r}} k_{L^{1}}(v) \quad C:
$$

Proof. Fix $x 2 E, 0<r \quad \operatorname{diam}(E)$, and let $E_{x ; r}=E \backslash B(x ; r) \backslash \quad x ; r$, where $x_{;} r=f p+A(p): p 2 P g$ is given by the BPLG condition, and Lip(A) $M$. Then, set $V_{x ; r}=P$ and $=[2(1+M)]^{1}$. If $\vee 2 B\left(V_{x ; r} ;\right)$ one can also write $x_{i} ;=f v+A_{V}(v): \vee 2 \vee g$, where $A_{V}: V ! V ?$ and $\operatorname{Lip}\left(A_{V}\right) \quad 3(1+M)$.

Suppose that $\mathrm{V}$ is as above, $\vee 2 \mathrm{~V}$ and $\mathrm{s}>0$. Then

$$
v^{1}(B v(v ; s)) \backslash \quad x ; r \quad B\left(v+A_{v}(v) ; 4(1+M) s\right) ;
$$

which implies that

$$
\frac{{ }^{v} H^{n} j_{E_{x ; r}}(B,(v ; s))}{H^{n}\left(B_{V}(v ; s)\right)} \cdot 1:
$$

The proposition now follows from Theorem 2.12 of [7].

Remark 1.5. We write $A$. $p$ B if $A \quad C B$ for some constant $C>0$ depending only on the parameter $p$; the notation A . B means that the constant $C$ is absolute, or depends only on parameters, which can be regarded as "fixed" in the situation. The two-sided inequality B . ${ }_{p} A, p B$ is abbreviated to A $p$. 
The main result of the paper asserts that the necessary condition for BPLG in Proposition 1.4 is also sufficient, and the uniform $L^{1}$-bound for the projections can even be relaxed to an averaged bound for the $L^{2}$-norms:

Theorem 1.6. Let $E \quad R^{d}$ bean $n-A D R$ set. Supposethat thereexist constants $>0$ and $C<1$ such that thefollowing holds. For every $\times 2 \mathrm{E}$ and $r 2(0 ; \operatorname{diam}(E)$ ], there is an $H^{n}$-measurablesubset $E_{x ; r} \quad E \backslash B(x ; r)$ and a subspace $V_{x ; r} 2 G(d ; n)$ with the following properties:

(1) $\mathrm{H}^{\mathrm{n}}\left(\mathrm{E}_{\mathrm{x} ; \mathrm{r}}\right) \quad \mathrm{r}^{\mathrm{n}}$,

(2) ${ }^{V}{ } H^{n} j_{E_{x ; r}} 2 L^{2}(V)$ for $d ; n-a . e V 2 B\left(V_{x ; r} ;\right)$, and

$$
\begin{aligned}
& \text { Z }
\end{aligned}
$$

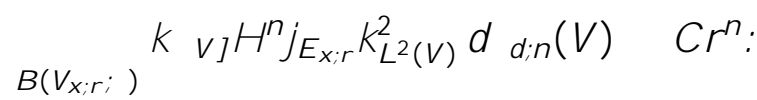

Then E has BPLG. In particular, E is n-UR.

We now discuss the context and history of the result. The Besicovitch-Federer projection theorem is a characterisation of rectifiability in terms of projections. Since the introduction of uniform rectifiability, it has been a natural question to find a quantitativeanalog of theBesicovitch-Federer result for UR sets. H owever, an unpublished example of T. Hrycak shows that uniform rectifiability does not imply quantitatively large projections, at least in the obvious sense: given $>0$, H rycak's construction produces a UR-set $E \quad R^{2}$, with constants independent of , such that $\mathrm{H}^{1}\left({ }_{L}(E)\right)$ for every line $L 2 \mathrm{G}(2 ; 1)$.

Theslightly stronger condition BPLG, however, does imply quantitatively large projections (this is well-known but also follows from Proposition 1.4), and so characterising BPLG in terms of projections seems to be a more natural question to ask. Perhaps the most obvious candidate for such a characterisation is through the big projections in plenty of directions (BPPD) assumption. An n-ADR set $E \quad R^{d}$ has BPPD, if there exists a constant > 0 with the following property: for every $x 2 \mathrm{E}$ and $\mathrm{r} 2(0 ; \operatorname{diam}(\mathrm{E})]$, there is an $n-p l a n e V_{x ; r} 2 \mathrm{G}(\mathrm{d} ; \mathrm{n})$ such that

$$
H^{n}(v(E \backslash B(x ; r))) \quad r^{n}
$$

for every $\vee 2$ B $\left(V_{x ; r} ;\right)$. Indeed, G. David and S. Semmes ask in $[2,4]$ whether BPLG is equivalent to BPPD. This remains open to date. It is clear that BPLG implies BPPD. In the converse direction, the quantitative Besicovitch projection theorem of T. Tao [9] yields some structural information about BPPD sets, but the conclusions are weaker than BPLG.

It is also known, see [4], that BPLG is characterised by a combination of BPPD and an extra hypothesis called the the weak geometric lemma - an additional regularity assumption not connected with projections. In contrast, our result is the first to characterise BPLG using projections, and projections only.

Let us briefly seehow the BPPD hypothesis is connected with our assumptions. To this end, suppose that a set $E^{0} \quad E \backslash B(x ; r)$ satisfies $H^{n}\left(E q \& r^{n}, v_{]} H^{n} j_{E} 02\right.$ 
$L^{2}(V)$ and $k v_{v j} H^{n} j_{E} o k_{L^{2}(V)}^{2}$. $r^{n}$ for some $V 2 G(d ; n)$. Then, $r^{2 n} \cdot k_{v j} H^{n} j_{E} o k_{L^{1}(V)}^{2} \quad H^{n}\left(v(E g) k_{v j} H^{n} j_{E} o k_{L^{2}(V)}^{2} \cdot H^{n}(v(E \backslash B(x ; r))) r^{n}\right.$; which implies (1.7) for this particular $V$. Therefore, for those $V$ in Theorem 1.6 such that $\mathrm{K}_{{ }_{\mathrm{V}}} \mathrm{H}^{\mathrm{n}} \mathrm{j}_{\mathrm{E}_{\mathrm{x} ; \mathrm{r}}} \mathrm{K}_{\mathrm{L}^{2}(\mathrm{~V})}$. $\mathrm{r}^{\mathrm{n}}$, our hypothesis is strictly stronger than (1.7); on the other hand, our "averaged" hypothesis is more relaxed than the uniform requirement of BPPD.

1.1. Outline of the proof. After a suitable translation, scaling and rotation, the proof of Theorem 1.6 reduces to verifying the following statement:

Theorem 1.8. Let $E_{0} \quad R^{d}$ bean $n-A D R$ set, and assumethat $E_{1} \quad E_{0} \backslash B(0 ; 1)$ is an $\mathrm{H}^{\mathrm{n}}$-measurable subset satisfying thefollowing two properties:

(i) $\mathrm{H}^{\mathrm{n}}\left(\mathrm{E}_{1}\right) \quad>0$, and

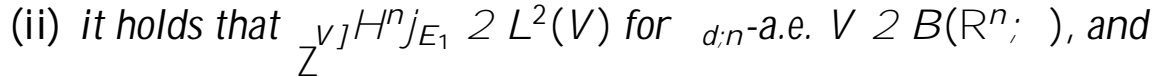

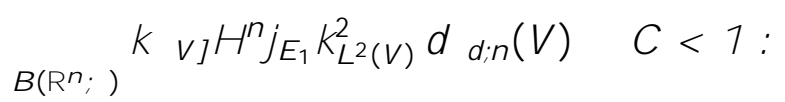

Then, thereexists a Lipschitz function $A: R^{n} ! R^{d}{ }^{n}$ such that Lip(A) . ;c 1 and the Lipschitz graph

$$
=f(x ; A(x)): x 2 R^{n} g
$$

satisfies

$$
\mathrm{H}^{\mathrm{n}}\left(\mathrm{E}_{1} \backslash\right) \&_{; \mathrm{C}} 1:
$$

The proof divides into one main lemma and one main proposition.

Definition 1.9 (Cones). For $x 2 R^{d}, \vee 2 G(d ; k)$ and $2(0 ; 1)$, we set

$$
X(x ; V ;)=f y 2 R^{d}: j V^{?}(x \quad y) j \quad j x \quad y j g:
$$

Given a cone $X(x ; V ; \quad)$ and two radii $0<r<R<1$, we write

$$
\mathrm{X}(\mathrm{x} ; \mathrm{V} ; \mathrm{R} ; \mathrm{r}):=\mathrm{X}(\mathrm{x} ; \mathrm{V} ;) \backslash[\mathrm{B}(\mathrm{x} ; \mathrm{R}) \mathrm{nU}(\mathrm{x} ; \mathrm{r})] \text {; }
$$

where $B(x ; R)$ and $U(x ; r)$ are, respectively, the closed and open balls of radii $R>0$ and $r>0$ centred at $x$. Note that $X(x ; V ; \quad ; R ; r)$ is a closed set for $0<$ $r<R<1$. Finally, writing $R^{d}=R^{n} \quad R^{d} n$, we use the shorthand notation $X(x ;):=X\left(x ; R^{d} n ;\right)$ and $X(x ; \quad R ; r):=X\left(X ; R^{d} n ; ; R ; r\right)$.

Our main lemma reads as follows:

Lemma 1.10. Assumethat $E_{0}$ and $E_{1}$ satisfy thehypotheses of Theorem 1.8. Then, there exist numbers $\mathrm{M}_{0}=\mathrm{M}_{0}(; \mathrm{C}) 2 \mathrm{~N}, 0=0(; \mathrm{d})>0$ and an $\mathrm{H}^{\mathrm{n}}$-measurable subset $E_{2} \quad E_{1}$ with thefollowing properties: $\mathrm{H}^{\mathrm{n}}\left(\mathrm{E}_{2}\right) ; \mathrm{c} 1$, and if $\times 2 \mathrm{E}_{2}$, then

$$
\# f j 2 Z: X\left(X ; 0 ; 2^{j} ; 2^{j}{ }^{1}\right) \backslash E_{2} G ; g \quad M \text { : }
$$

Before stating the main proposition, we need another definition: 
Definition 1.11. A set $E \quad R^{d}$ satisfies the n-dimensional ( ; $M$ )-property, if

$$
\# f j 2 Z: X\left(x ; ; 2^{j} ; 2^{j}{ }^{1}\right) \backslash E g \quad M
$$

for all $\times 2 \mathrm{E}$.

Remark 1.12. Observe that if $E$ satisfies the ( ; $M$ )-property with $M=0$, then $E$ is entirely contained in a Lipschitz graph with Lipschitz constant $1=$. Indeed, there holds that

$$
j R^{n}\left(\begin{array}{llll}
x & y
\end{array}\right) j \quad j x \quad y j ; \quad x ; y 2 E \text { : }
$$

In particular, the restriction $\mathrm{R}^{\mathrm{n}} \mathrm{j}_{\mathrm{E}}$ of the orthogonal projection $\mathrm{R}^{\mathrm{n}}$ to $\mathrm{E}$ is one-toone, and onecan definea $(1=)$-Lipschitz inversef : $\mathrm{R}^{n}(E)$ ! E. By Kirszbraun's theorem, see Theorem 2.10.43 in [6], this can be extended to a Lipschit mapping $f \because R^{n} ! R^{d}$ with $\operatorname{Lip}(f) \quad 1=$, and then

$$
\text { E } f(y ; A(y)) \text { : y } 2 R^{n} g ;
$$

where $A: R^{n} ! R^{d} n$ is defined by $A=R^{d} n \quad f \sim$. This is essentially theargument from Lemma 15.13 in Mattila's book [7].

In the language of Definition 1.11, Lemma 1.10 claims that $E_{2}$ satisfies the $n-$ dimensional $\left(0 ; \mathrm{M}_{0}\right)$-property for some $0 ; \mathrm{M}_{0}$ depending only on and $\mathrm{C}$. Here is the main proposition:

Proposition 1.13. Assume that $E_{0}$ is $n-A D R$, and assumethat $E_{2} \quad E_{0} \backslash B(0 ; 1)$ is an $\mathrm{H}^{\mathrm{n}}$-measurablesubset with $\mathrm{H}^{\mathrm{n}}\left(\mathrm{E}_{2}\right)$;c 1 and satisfying then-dimensional $(; \mathrm{M})$ property for some $>0$ and $M \quad 0$. Then, there is an $\mathrm{H}^{\mathrm{n}}$-measurable subset $\mathrm{E}_{3} \quad \mathrm{E}_{2}$ with $\mathrm{H}^{\mathrm{n}}\left(\mathrm{E}_{3}\right) \quad ; \mathrm{C} ; \mathrm{M} ; \quad 1$ and satisfying the $(\Rightarrow ; 0)$-property. Hereb 1 is a constant depending only on $\mathrm{d}$.

Taking Lemma 1.10 and Proposition 1.13 for granted, it is straightforward to complete the proof of Theorem 1.8:

Proof of Theorem 1.8. Use Lemma 1.10 to find 0 and $M_{0}$, and the set $E_{2} \quad E_{1}$ satisfying then-dimensional ( $0 ; \mathrm{M}_{0}$ )-property. Then, use Proposition 1.13 to find $E_{3} \quad E_{2}$ with $\mathrm{H}^{\mathrm{n}}\left(\mathrm{E}_{3}\right)$;c 1 and satisfying the n-dimensional ( $0 \Rightarrow$; 0 )-property. Now, $E_{3}$ is contained in a Lipschitz graph with Lipschitz constant $b={ }_{0}$ by Remark 1.12, and

$$
H^{n}\left(E_{1} \backslash\right) \quad H^{n}\left(E_{3}\right) \& ; c 1:
$$

This completes the proof.

Acknowledgements. Wethank the refereefor various suggestions, which hel ped to improve the readability of the paper. 


\section{Proof of The MAIN LEMma}

We start by proving an easy but very useful auxiliary lemma:

Lemma 2.1. Let $E_{0}$ bean $n-A D R$ set with $H^{n}\left(E_{0}\right) \quad c>0$, le $E_{1} \quad E_{0} \backslash B(0 ; 1)$ be an $\mathrm{H}^{\mathrm{n}}$-measurable subset, and let

$$
E_{1 ;}:=f \times 2 E_{1}: H^{n}\left(E_{1} \backslash B\left(x ; r_{x}\right)\right) \quad r_{x}^{n} \text { for someradius } 0<r_{x} \quad \text { lg: }
$$

Then $H^{n}\left(E_{1 ;}\right)$. with the bound depending only on $c$ and theADR constant of $E_{0}$.

Proof. The set $E_{1 ;}$ is covered by the balls $B\left(x ; r_{x}=5\right), x 2 E_{1 ;}$, so the $5 r$-covering lemma can be used to extract a disjoint subcollection $f B\left(x_{i} ; r_{x_{i}}=\right) g_{i 2 N}$ with the property that the balls $f B\left(x_{i} ; r_{x_{i}}\right) g_{i 2 N}$ cover $E_{1 ;}$. Let $C_{0}>5$ be so large that $r_{x_{i}}=C_{0} \quad \operatorname{diam}\left(E_{0}\right)$ (since $r_{x_{i}} \quad 1$ uniformly, $C_{0}$ depends only on $c$ and the ADR constants of $\left.E_{0}\right)$. N ow, we have that

$$
\begin{aligned}
H^{n}\left(E_{1 ;}\right) & { }_{i 2 N} H^{n}\left(E_{1} \backslash B\left(x_{i} ; r_{x_{i}}\right)\right) \\
& { }_{i 2 N} H^{n}\left(E_{0} \backslash B\left(x_{i} ; r_{x_{i}}=C_{0}\right)\right) \\
& H^{n}\left(E_{0} \backslash B(0 ; 2)\right) . ;
\end{aligned}
$$

as claimed.

Of course, the lemma cannot be used to conclude that theset $E_{1} n E_{1 ;}$ is $n-A D R$, but it is still somewhat more regular than $E_{1}$, and and this will be useful in the following proofs.

We also need another technical lemma:

Lemma 2.2. Fix ; $>0$ and le $W 2 \mathrm{G}(\mathrm{d} ; \mathrm{n})$. Assume that $z 2 \mathrm{R}^{\mathrm{d}}$ satisfies fizj < $\min (0 ;=2)$, where 0 is a small constant depending only on $d$, and $j w z j \quad 0 j z j$ for a small enough $0=0(n ;)>0$. Define $B_{z}=f \vee 2 G(d ; n): j \vee z j \quad g$. Then,

$$
A(z):={ }_{d ; n}\left(B_{z} \backslash B_{G(d ; n)}(W ; \quad) \& \quad \overline{j z j}^{n}:\right.
$$

We postpone the proof to A ppendix A.

Proof of Lemma 1.10. In what follows, the ADR constants of $E_{0}$ and the constants and $C$ from the statement of Lemma 1.10 will be treated as "fixed" in the sense that ". ;c" is abbreviated to ". ". We begin by applying Lemma 2.1 twice. First, with $H^{n}\left(E_{1}\right)$, we remove $E_{1}$; from $E_{1}$ : thus, for a suitable 1 , the set $E^{0}:=E_{1} \mathrm{nE}_{1}$; satisfies $\mathrm{H}^{\mathrm{n}}(\mathrm{E}) \quad 1$ and has the property that if $\times 2 \mathrm{E}^{0}$ and $0<r \quad 1$, then

$$
H^{n}\left(E_{1} \backslash B(x ; r)\right) \quad r^{n}:
$$

Then, we apply the lemma again to $E^{0}$, this time with ${ }^{0} \quad H^{n}(E)$, to the effect that the set $E:=E^{0} n E_{0}^{0}$ still satisfies $H^{n}(E) \quad 1$, and if $\times 2 E, 0<r \quad 1$, then

$$
H^{n}\left(E^{O} \backslash B(x ; r)\right) \quad r^{n}:
$$


Let $0=0=2$, where $0>0$ appears in Lemma 2.2 with $=$. Given $M>0$ let $E^{M}$ consist of those $\times 2$ for which thereareat least $M$ scales $2{ }^{j}, j 2 Z$, such that

In symbols,

$$
X\left(X ; 0 ; 2^{j} ; 2^{j}{ }^{1}\right) \backslash E G ;:
$$

$$
E^{M}=f \times 2 E: \# f j 2 Z: X\left(X ; 0 ; 2^{j} ; 2^{j}{ }^{1}\right) \backslash E G ; g \quad M g:
$$

In a moment, we will show that $\mathrm{H}^{\mathrm{n}}\left(\mathrm{E}^{\mathrm{M}}\right) .1 \neq \mathrm{M}$; this completes the proof, because then, for a large enough $M$, the set $E_{2}=E n E^{M}$ will be the set we were looking for.

We start with a preliminary reduction. Let $C_{0} \quad 1$ be a large constant depending only on , to be specified later. Observe that for every $\times 2 \mathrm{E}^{\mathrm{M}}$, there is a constant $x>0$ such that there are at least $M$ scales $2{ }^{j} \quad C_{0} \times$ satisfying (2.5). If $E^{\mathrm{M} ;}=f \times 2 E^{\mathrm{M}}: \times \mathrm{g}$, we can choose $>0$ so small that $H^{n}\left(E^{M} ;\right) \quad H^{n}\left(E^{M}\right)=2$. In particular, it suffices to show that $H^{n}\left(E^{M} ;\right) . \quad 1 \neq M$, where the implicit constant does not depend on . This is what we will do, but in order to avoid obscuring the notation any further, we assume that $E^{M}=E^{M}$; ; note that (2.3) and (2.4) are obviously unaffected by the passage from $E^{M}$ to $E^{M}$; .

With as in the previous paragraph, let $\mathrm{F}^{\mathrm{M}}$ and $\mathrm{F}^{0}$ be maximal -separated sets inside $E^{M}$ and $E^{0}$, respectively; since $E^{M} \quad E^{0}$, we can also arrange so that $\mathrm{F}^{\mathrm{M}} \quad \mathrm{F}^{0}$. We wish to find lower and upper bounds on the amount of triples $(x ; y ; V)$, where $x ; y 2 F^{0}, V 2 B\left(R^{n} ;\right)$, and

$$
\mathrm{j} v\left(\begin{array}{lll}
x & y
\end{array}\right) \mathrm{j}:
$$

The idea is that assumption (ii) of Theorem 1.8 will give us an upper bound for such triples, whereas a lower bound can be obtained, via (2.5), by choosing $\times 2$ $\mathrm{F}^{\mathrm{M}} \quad \mathrm{F}^{0}$ and $\mathrm{y} 2 \mathrm{~F}^{0}$.

We start with the lower bound. Note that

$$
\# F^{M} \& H^{n}\left(E^{M}\right) \quad{ }^{n} ;
$$

because $E^{M}$ is covered by the balls $B(x ; 2), x 2 F^{M}$, and the AD-regularity of $E_{0} \quad E^{M}$ implies that $H^{n}\left(B(x ; 2) \backslash E^{M}\right)$. ${ }^{n}$. Fix $x 2 F^{M}$ for the moment. Now, let $2{ }^{j} \quad C_{0}$ be one of the scales such that (2.5) holds, and choose a point

$$
y_{j} 2 x\left(x ; 0 ; 2^{j} ; 2^{j}{ }^{1}\right) \backslash E \text { : }
$$

First, choose $c_{0}=c_{0}\left({ }_{0}\right)>0$ so small that we have

$$
B\left(y_{j} ; c_{0} 2^{j}\right) \quad X\left(x ; 2 ; 0 ; 2^{j+1} ; 2^{j}{ }^{2}\right) \text { : }
$$

This is depicted in Figure 1 . Since $y_{j} 2 E$, we infer from (2.4) that

$$
H^{n}\left(E^{9} \backslash B\left(y_{j} ; C_{0} 2^{j}{ }^{1}\right)\right) \& 2^{j n}:
$$

Choosing $\mathrm{C}_{0}=\mathrm{C}_{0}\left(\right.$ ) so large that $\mathrm{C}_{0} \quad 2=\mathrm{C}_{0}$ we have that

$$
E^{0} \backslash B\left(y_{j} ; c_{0} 2^{\left.j{ }^{1}\right)} \underset{w 2 F 9 B\left(y_{j} ; c_{0} 2 j\right)}{B(w ; 2):}\right.
$$




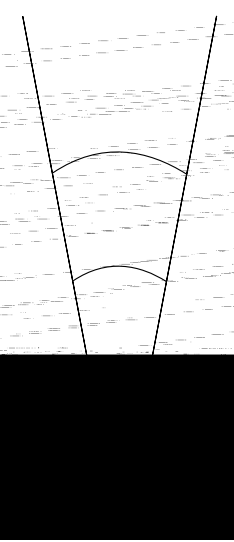

FIGURE 1. Finding the balls $B\left(y_{j} ; C^{j}\right) \quad X\left(x ; 2^{j} ; 2^{j+1} ; 2^{j}{ }^{2}\right)$.

To see this, notice that if $u 2 E^{0} \backslash B\left(y_{j} ; c_{0} 2^{j}{ }^{1}\right)$, there is $w 2 F^{0}$ so that jw uj $2{ }^{\mathrm{j}} \mathrm{C}_{0}{ }^{1} \quad \mathrm{C}_{0} 2^{\mathrm{j}}{ }^{1}$. Wenow conclude that

$$
\#\left(\mathrm{~F}^{0} \backslash B\left(\mathrm{y}_{\mathrm{j}} ; \mathrm{c}_{0} 2^{\mathrm{j}}\right)\right) \&{ }^{\mathrm{n}_{2}}{ }^{\mathrm{j}} \text {; }
$$

again by the $A D$-regularity of $E_{0}$. In order to use Lemma 2.2 we enlarge $C_{0}=$

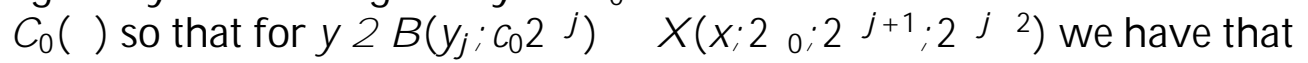

$$
\text { jx yj } 2^{j} 2^{2} \quad \frac{1}{4} C_{0} \quad \overline{\min (0 ; \quad=2)} \text { : }
$$

Hence, Lemma 2.2 applies to $z=x \quad y\left(\right.$ sincej $R^{n}(z) j \quad 2$ ojzj $={ }_{0}$ jzj), and so

$$
\begin{aligned}
& X \\
& \text { y2F } 9 \text { B }\left(y_{j} ; c_{0} 2 j\right) \\
& d ; n\left(B_{x} y \backslash B\left(R^{n} ;\right)\right) \& \quad{ }^{n} 2^{j n} \overline{2 j}^{n}=1 ;
\end{aligned}
$$

where $B_{x} y=B_{z}$ was defined in Lemma 2.2. Then, varying the scale $2^{j}$, hence the point $y_{j}$, we find $\& M$ disjoint balls $B\left(y_{j} ; c_{0} 2^{j}\right)$ inside $X(x ; 2$ o like above, and therefore

$$
\operatorname{y2F}^{X \times(x ; 20)} d ; n\left(B_{x} y \backslash B\left(R^{n} ;\right)\right) \& M:
$$

This finally yields that

$$
\begin{aligned}
& X \quad X \quad X \\
& x ; y_{2} F^{0} \quad x^{2} F^{M} \text { y2F } 9 x(x ; 20) \\
& \& M \quad H^{n}\left(E^{M}\right) \quad n \text { : }
\end{aligned}
$$

We then go for the upper bound. For $V 2 G(d ; n)$ define $_{V}: V ! R$ by setting

$$
f_{V}(z)={ }_{x 2 F^{0}}^{X} I_{B(v x ;)}(z):
$$


Notice that

$$
\begin{aligned}
& f_{v}(z)^{2} d H^{n}(z)={ }^{x ; y 2 F^{0}} v^{Z} I_{B(v x ;)} B_{(v y ;)}(z) d H^{n}(z) \\
& \&{ }^{n} \# f(x ; y) 2 F^{0} \quad F^{0}: j v(x \quad y) j \quad g:
\end{aligned}
$$

Therefore, we now have that

$$
\begin{aligned}
& H^{n}\left(E^{M}\right) M . n^{X} \quad{ }_{d ; n}\left(B_{x} y \backslash B\left(R^{n} ;\right)\right) \\
& \mathrm{Z} ; \mathrm{Y}^{2 \mathrm{~F}} \mathrm{O} \\
& ={ }_{Z}{ }_{Z}^{B\left(R^{n} \dot{Z}\right)} \quad \# f(x ; y) 2 F^{0} \quad F^{0}: j \vee(x \quad y) j \quad g d_{d ; n}(V) \\
& B\left(R^{n} ;\right) \quad f_{V}(z)^{2} d H^{n}(z) d d_{d ; n}(V) \text { : }
\end{aligned}
$$

Recall that if $x 2 F^{0} \quad E^{0}$, then $H^{n}\left(E_{1} \backslash B(x ;)\right) \quad n$ by (2.3). Using this we estimate $f_{v}$ pointwise:

$$
\begin{aligned}
& f_{V}(z) \quad n \quad X \quad H^{n}\left(E_{1} \backslash B(x ;)\right) \\
& x 2 \mathrm{~F} 0 \\
& j \vee x \quad z j \\
& { }^{n} H^{n}\left(E_{1} \backslash \quad v^{1}\left(B_{V}(z ; 2)\right)\right) . M_{V}\left(v_{J} H^{n} j_{E_{1}}\right)(z):
\end{aligned}
$$

Here $M_{V}$ is the Hardy-Littlewood maximal function on $V$. The operator $M_{V}$ is bounded on $L^{2}(V)$, see Theorem 1 on $p .13$ in Stein's book [8]. Therefore, we can conclude the proof as follows, using assumption (ii) from Theorem 1.8:

Z

$$
H^{n}\left(E^{M}\right) M . \quad B_{B\left(R^{n} ;\right)} k_{v]} H^{n} j_{E_{1}} k_{L 2(V)}^{2} d_{d ; n}(V) .1:
$$

\section{PROOF OF THE MAIN PROPOSITION}

Recall the notation for general cones from Definition 1.9. When $\mathrm{V}=\operatorname{span}(\mathrm{w}) 2$ $\mathrm{G}(\mathrm{d} ; 1)$, w $2 \mathrm{~S}^{\mathrm{d}} 1$, we introduce shorthand notation for one-dimensional onesided cones, namely

$$
X^{+}(x ; w ;):=X(x ; \operatorname{span}(w) ;) \backslash \text { fy } 2 R^{d}:(y \quad x) \text { w } 0 g:
$$

The restricted version $X^{+}(x ; w ; \quad R ; r), 0<r<R<1$, is defined in the obvious way. We start with a lemma, which states that cones of arbitrary co-dimension with a fixed aperture can be covered by a bounded number of one-dimensional one-sided cones - even ones with a slightly smaller aperture: 
Lemma 3.1. Fix $2(0 ; 1)$, s $2(0 ; 1]$ and $V 2 G(d ; k)$. Then, there exist vectors $\mathrm{w}_{1} ;:: ; ; \mathrm{w}_{\mathrm{m}} 2 \mathrm{~S}^{\mathrm{d}}{ }^{1}, \mathrm{~m} .(\mathrm{s})^{1 \mathrm{~d}}$, such that

$$
X\left(0 ; V_{i}\right) \quad_{j=1}^{[m} X^{+}\left(0 ; w_{j} ; s\right){ }_{j=1}^{[m} X^{+}\left(0 ; w_{j} ;\right) \quad X(0 ; V ; b):
$$

Hereb 1 is a constant depending only on $\mathrm{d}$.

Proof. It suffices to find $w_{1} ;:: ; ; w_{m} 2 S^{d}{ }^{1}$ such that (3.2) holds with all the cones intersected with $S^{d}{ }^{1}$. Find an $(s)-n e t w_{1} ;:: ; w_{m} \quad X\left(0 ; V_{;} ;\right) \backslash S^{d}{ }^{1}$. Then $\mathrm{m}$. $(\mathrm{s})^{1}{ }^{\mathrm{d}}$. Now, write $\mathrm{W}_{\mathrm{j}}:=\mathrm{w}_{\mathrm{j}}^{\text {, }}$, fix y $2 \times(0 ; \mathrm{V} ;) \backslash \mathrm{S}^{\mathrm{d}}{ }^{1}$, and pick $w_{j}$ such that jy $w_{j} j \quad s$. Then $y \quad w_{j} \quad 0$ and

$$
\text { j } w_{j}(y) j=j \quad w_{j}\left(y \quad w_{j}\right) j \quad s ;
$$

which proves that y $2 X^{+}\left(0 ; w_{j} ; s\right) \backslash S^{d}{ }^{1}$ and hence the first inclusion of (3.2). To provethesecond inclusion, fix 1 j $\operatorname{mand} z 2 X^{+}\left(0 ; w_{j} ;\right) \backslash S^{d}{ }^{1}$. Observe that jz $\quad w_{j} j \quad b^{0}$ for some constant $b^{0}=b(d)$, whence

$$
\text { j } v^{?}(z) j \quad j \quad v^{?}\left(\begin{array}{ll}
z & w_{j}
\end{array}\right) j+j \quad v^{?}\left(w_{j}\right) j \quad j z \quad w_{j} j+\quad\left(b^{0}+1\right):
$$

This proves the lemma with $b=b^{0}+1$.

Lemma 3.1 allows us to reduce the proof of the main Proposition 1.13 to the "co-dimension 1" case. The relevant (one-dimensional, one-sided) cones in this co-dimension are directed, which is quite useful in the proof.

Proposition 3.3 (Main proposition in co-dimension 1). Let w $2 \mathrm{~S}^{d} 1$. Assumethat an $\mathrm{H}^{\mathrm{n}}$-measurablese $\mathrm{F} \quad \mathrm{R}^{\mathrm{d}}$ satisfies the following conditions:

(a) $H^{n}(F)=: \quad>0$, and $F \quad E_{0} \backslash B(0 ; 1)$ for somen-ADR set $E_{0}$,

(b) for some $>0, M 2 \mathrm{~N}$, and for every point $\times 2 \mathrm{~F}$ thereholds that

$$
\# f j 2 \mathrm{Z}: \mathrm{X}^{+}\left(\mathrm{X} ; \mathrm{w} ; 2^{\mathrm{j}} ; 2^{\mathrm{j}^{1}}\right) \backslash \mathrm{F} \in ; \mathrm{g} \quad \mathrm{M} \text { : }
$$

Weabbreviate(a) and (b) by saying that that $F$ satisfies the $(; M ; w)$-property. Then, if $M \quad 1$ and $>0$ is small enough (depending on only on d), thereexists a compact set K $\quad F$ with $H^{n}(K) \quad ; 1$, which satisfies the ( $=2 ; M \quad 1 ;$ w)-property.

The general idea of the argument is to write down an explicit algorithm, which refines $\mathrm{F}$ by deleting some points in several stages, but all the time keeps track that not too much is wasted. When the algorithm eventually stops, it will output the desired set $\mathrm{K}$. Before giving the details, let us see how the general version of the main proposition follows from Proposition 3.3.

Proof of Proposition 1.13. Assumethat $E_{2} \quad E_{0}$ satisfies then-dimensional $\left(0 ; M_{0}\right)$ property. Then, with $V=\left(R^{n}\right)^{\text {? }}$,

$$
\# f j 2 Z: X\left(x ; V ; 0 ; 2^{j} ; 2^{j}{ }^{1}\right) \backslash E_{2} G ; g \quad M_{0}
$$


for all $\times 2 E_{2}$. Now, use Lemma 3.1 with $={ }_{0} \Rightarrow b$ and $s=2 M_{0}$ to find vectors $\mathrm{w}_{1} ;::: ; \mathrm{w}_{\mathrm{m}} 2 \mathrm{~S}^{1}, \mathrm{~m} .\left(\mathrm{o}_{0}=\left(2^{\mathrm{M}}{ }^{\mathrm{ob}}\right)\right)^{1} \mathrm{~d}^{\mathrm{d}}$, such that

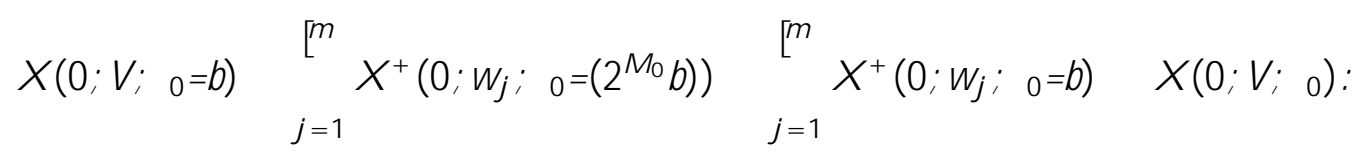

It follows from translation invariance that

$$
X\left(x ; V_{;} \quad 0=b, 2^{j} ; 2^{j}{ }^{1}\right){ }_{j=1}^{[m} X^{+}\left(x ; w_{j} ; 0=\left(2^{M} 0 b\right) ; 2^{j} ; 2^{j}{ }^{1}\right)
$$

for all $\times 2 R^{d}$ and $j 2 Z$. Further, $E_{2}$ satisfies the $\left({ }_{0}=b_{1} M_{0} ; W_{j}\right)$-property from Proposition 3.3 for all 1 j $m$. Thus, iterating that proposition $M_{0}$ times for each 1 j $m$, hence $\mathrm{mM}_{0}$ times al together, one finds a set $E_{3} \quad E_{2}$ satisfying the $\left.\left({ }_{0}=2^{M^{0}} b\right) ; 0 ; w_{j}\right)$-property for all $1 j \quad j$. It follows from (3.4) that $E_{3}$ satisfies the n-dimensional ( $0 \neq 0 ; 0)$-property, and Proposition 1.13 is proved.

Proof of Proposition 3.3. We assume that $w=e_{d}=(0 ; 0 ;::: ; 0 ; 1)$. Before starting to describe the algorithm to find $K \quad F$, we make two easy reductions: first, without loss of general ity, we may assume that if $\times 2 \mathrm{~F}$ and

$$
\mathrm{X}^{+}\left(\mathrm{X} ; \mathrm{w} ; ; 2^{\mathrm{j}} ; 2^{\mathrm{j}}{ }^{1}\right) \backslash \mathrm{FG} \text {; }
$$

then $2^{j} \quad$ for some small constant $>0$. Simply, for every $\times 2 \mathrm{~F}$, there is some $\times>0$ with this property, and then we can take $>0$ so small that $H^{n}(F 9) H^{n}(F)=2$, where $F^{0}:=F n f \times 2 F: \times<$. After this, we would proceed with the proof as below, only replacing $\mathrm{F}$ by $\mathrm{F}$. Second, we may assume that $F$ is compact; otherwise we can always find a compact subset of $F$ (or $F$ with almost the same $\mathrm{H}^{\mathrm{n}}$-measure, and then we can find $\mathrm{K}$ inside this subset as below.

We now begin to describe the algorithm. The following points (I)-(IV) summarise the key features.

(I) There will be a sequence of compact sets $F=F^{0} \quad F^{1} \quad F^{2}$, where $F^{k+1}$ is obtained from $F^{k}$ by deleting a certain open set $D^{k}$.

(II) Thus, there will also be a sequence of deted sets $D^{k} \quad F^{k}, k 2$ f $0 ; 1 ;::$ :g.

(III) There will be a sequence of saved sets $S^{k} \quad F^{k} \quad F, k=f 0 ; 1 ;:: g$, which are disjoint from each other and all the deleted sets $D^{i}, i \quad k^{1}$ satisfy

$$
\mathrm{H}^{\mathrm{n}}\left(\mathrm{S}^{\mathrm{k}}\right) \& \text { maxf } \mathrm{H}^{\mathrm{n}}\left(\mathrm{D}^{\mathrm{k}}\right) ;{ }^{\mathrm{n}} \mathrm{g} \text {; }
$$

and have the property that if

$$
\times 2 \underset{i k}{[} S^{i}
$$

${ }^{1}$ The sets $S^{k}$ are also disjoint from the deleted sets $D^{i}$ with $i<k$, as $S^{k} \quad F^{k}=F n_{i<k} D^{i}$. 
then there are at most $M \quad 1$ scales $2^{j}$ such that

$$
\mathrm{X}^{+}\left(\mathrm{x} ; \mathrm{w} ;=2 ; 2^{\mathrm{j}} ; 2^{j}{ }^{1}\right) \backslash \mathrm{i}_{\mathrm{i}}^{\mathrm{i}} \boldsymbol{\mathrm { k }} ;:
$$

(IV) We describe the structure of the saved sets. Let $\mathrm{F}^{\mathrm{k} ; \mathrm{M}}$ be the set of points in $\mathrm{F}^{\mathrm{k}}$ such that there are exactly $\mathrm{M}$ scales $2^{\mathrm{j}}$ such that

$$
\mathrm{X}^{+}\left(\mathrm{X} ; \mathrm{w} ; \quad=2 ; 2^{\mathrm{j}} ; 2^{\mathrm{j}}{ }^{1}\right) \backslash \mathrm{F}^{\mathrm{k}} \mathrm{G} ; \text { : }
$$

A point $x 2 \mathrm{~F}^{\mathrm{k}}$ is then called $\mathrm{k}$-bad, if $x 2 \mathrm{~F}^{\mathrm{k} ; \mathrm{M}}$, and furthermore

$$
H^{n}\left(B(x ; r) \backslash F^{k ; M}\right) \quad r^{n}
$$

for all radii $0<r \quad 1$, where $\quad H^{n}(F)$ is a constant to be specified in Stopping condition 3.6 below. Using the compactness of $F^{k}$ and the uniform lower bound for thenumbers $2{ }^{j}$, it is easy to verify that the set of k-bad points is compact. Thus, if there are any k-bad points to begin with, there exists a (possibly non-unique) $k$-bad point $x_{k}$ with the smallest last coordinate $x_{k}^{d}$. With such a choice of $x_{k}$, the saved set $S^{k}$ will be defined as $B\left(x_{k} ; r_{k}\right) \backslash F^{k ; M}$ for some suitable radius $r_{k} \&$.

Note that if $x$ is $k$-bad and $k \quad 1$, then $x$ is also ( $\left.\begin{array}{ll}k & 1\end{array}\right)$-bad, simply because $F^{k} \quad F^{k}{ }^{1}$ and $F^{k ; M} \quad F^{k}$ 1;M . This implies, by the definition of $x_{k}$, that the last coordinates of the points $\mathrm{x}_{0} ; \mathrm{x}_{1} ;::: ; \mathrm{x}_{\mathrm{k}}$ form a non-decreasing sequence.

Finally, to every set $S^{k}=B\left(x_{k} ; r_{k}\right) \backslash F^{k ; M}$ we associate a somewhat larger set $B^{k}:=B\left(x_{k} ; 100 r_{k}\right) \backslash F^{k}$, which will have the property that if $x 2 B^{k}$, then there are at most $M \quad 1$ scales $2{ }^{j}$ such that

$$
\mathrm{X}^{+}\left(\mathrm{x} ; \mathrm{w} ; \quad 2 ; 2^{\mathrm{j}} ; 2^{\mathrm{j}}{ }^{1}\right) \backslash \mathrm{F}^{\mathrm{k}+1} \mathrm{G} ; \text { : }
$$

There will be two different stopping conditions, which bring the algorithm to a halt and output the desired set $\mathrm{K}$.

Stopping condition 3.5. Assume that the sets $D^{0} ;::: ; D^{k}$ and $S^{0} ;:: ; ; S^{k}$ have been defined, and either

or

$$
\underbrace{k}_{i=0} H^{n}\left(D^{i}\right) \quad H^{n}(F)=2
$$

$X^{k}$

$$
H^{n}\left(S^{i}\right) \quad H^{n}(F)=2:
$$

In both cases, we set

$$
K:={ }_{i k}^{[} S^{i}:
$$

By (III), the set K satisfies the requirements of Proposition 3.3, and the proof is complete. 
Stopping condition 3.6. Assume that the set $\mathrm{F}^{\mathrm{k}}$ has been defined, and satisfies $H^{n}\left(F^{k}\right) \quad H^{n}(F)=2$, and that the set of $k$-bad points, as in (IV), is empty. Thus, for every $\times 2 \mathrm{~F}^{\mathrm{k} ; \mathrm{M}}$, we have

$$
H^{n}\left(F^{k ; M} \backslash B\left(x ; r_{x}\right)\right) \quad r_{x}^{n}
$$

for some radius $0<r_{x} \quad 1$. Now choose $\quad H^{n}(F) \quad 1$ so small that, using Lemma 2.1, we have $H^{\mathrm{n}}\left(\mathrm{F}^{\mathrm{k} ; \mathrm{M}}\right) \quad \mathrm{H}^{\mathrm{n}}(\mathrm{F})=4$. We set

$$
\mathrm{K}:=\mathrm{F}^{\mathrm{k}} \mathrm{nF^{ \textrm {k } } \mathrm { M }} \text { : }
$$

Then, $H^{n}(K) \quad H^{n}\left(F^{k}\right) \quad H^{n}\left(F^{k ; M}\right) \quad H^{n}(F)=4$, and for every $\times 2 K$ there are at most $M \quad 1$ scales $2{ }^{j}$ such that

$$
\mathrm{X}^{+}\left(\mathrm{x} ; \mathrm{w} ; \quad 2 ; 2^{\mathrm{j}} ; 2^{\mathrm{j}}{ }^{1}\right) \backslash \mathrm{K} G ;:
$$

Thus, $\mathrm{K}$ satisfies the requirements of Proposition 3.3 , and the proof is complete.

Remark 3.7. N otice that, since $\mathrm{H}^{\mathrm{n}}\left(\mathrm{S}^{\mathrm{k}}\right) \&{ }^{\mathrm{n}}$ for every $\mathrm{k}$, the first stopping condition will be reached in .

${ }^{n}$ steps (unless the second stopping condition was reached before that). In particular, the algorithm terminates and outputs $\mathrm{K}$ after finitely many steps.

Next, we will explicitly describe how to construct the various sets $F^{k}, S^{k}$ and $D^{k}$. Define $S^{1}=;, D^{1}=$; and $F^{0}:=F$. Assume that $k \quad 0$ and the sets $\mathrm{F}^{0} ;:: ; ; \mathrm{F}^{\mathrm{k}}, \mathrm{D}^{1} ;::: ; \mathrm{D}^{\mathrm{k}}{ }^{1}$ and $\mathrm{S}^{1} ;::: ; \mathrm{S}^{\mathrm{k}}{ }^{1}$ have al ready been defined, and satisfy the properties listed in (I)-(IV); in particular, also the balls $B^{i}, i<k$, have been defined. Assume that the first stopping condition is not satisfied; otherwise the algorithm terminates and the proof is complete. In particular,

$$
H^{n}\left(F^{k}\right) \quad H^{n}(F) \quad{ }_{i<k}^{X} H^{n}\left(D^{i}\right) \quad H^{n}(F)=2 \text { : }
$$

N ext, assume that the second stopping condition is not satisfied; because of (3.8), this means that the set of k-bad points is non-empty, and - as required by (IV) we find one of them, $x_{k}$, with minimal last coordinate. Let $2{ }^{j_{k}}$ be one of the $M$ scales such that

$$
\mathrm{X}^{+}\left(\mathrm{X}_{k} ; \mathrm{w}_{2}=2 ; 2^{\mathrm{j}_{k}} ; 2^{\mathrm{j}_{k}}{ }^{1}\right) \backslash \mathrm{F}^{\mathrm{k}} \boldsymbol{G} ;:
$$

Let $r_{k}:=c 2 j_{k}$ for a suitable small $c=c(d ; \quad)>0$ to be specified later, and set

$$
S^{k}:=B\left(x_{k} ; r_{k}\right) \backslash F^{k ; M} ; \quad B^{k}:=B\left(x_{k} ; 100 r_{k}\right) \backslash F^{k} ;
$$

as required by (IV). Then

$$
H^{n}\left(S^{k}\right) \& 2^{j_{k} n} \quad{ }^{n} ;
$$

by the definition of $k$-badness. Furthermore, $S^{k}$ is disjoint from all the previous sets $S^{i}, i<k$, and even the larger sets $B^{i}, i<k$, because $S^{k} F^{k ; M}$, but if $\times 2 B^{i}$, then

$$
\mathrm{X}^{+}\left(\mathrm{x} ; \mathrm{w} ; \quad=2 ; 2^{\mathrm{m}} ; 2^{\mathrm{m}^{1}}\right) \backslash \mathrm{F}^{\mathrm{k}} \mathrm{G} ;
$$


can only hold for $M \quad 1$ scales $2 \mathrm{~m}$ by (IV). Next, we define the deleted set $D^{k}$ by

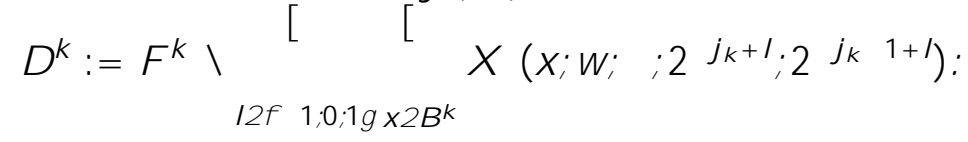

Here $\mathrm{X}$ stands for the interior of the cone $\mathrm{X}^{+}$(we want the deleted set to be relatively open in $\mathrm{F}^{\mathrm{k}}$ ). Then $\mathrm{D}^{\mathrm{k}}$ is contained in a single ball of radius . $2^{\mathrm{j} k}$, so $H^{n}\left(D^{k}\right) .2{ }^{j_{k} n}$. Combining this with (3.10), we see that

$$
H^{n}\left(S^{k}\right) \& \operatorname{maxf} H^{n}\left(D^{k}\right) ;{ }^{n} g ;
$$

as required in (III).

To complete the proof, we still need to show the disjointness of $D_{k}$ from the previous saved sets $\mathrm{S}^{i}, \mathrm{i}<\mathrm{k}$, the latter claim in (III) about ${ }_{i}{ }_{k} \mathrm{~S}^{i}$, and the claim about the set $B^{k}$ at the end of (IV). We begin with the last and easiest task. By the definition of $2 j_{k}$ in (3.9), there exists a point

$$
\mathrm{Z}_{\mathrm{k}} 2 \mathrm{X}^{+}\left(\mathrm{x}_{\mathrm{k}} ; \mathrm{w} ; \quad=2 ; 2^{\mathrm{j}_{\mathrm{k}}} ; 2^{\mathrm{j}_{\mathrm{k}}}{ }^{1}\right) \backslash \mathrm{F}^{\mathrm{k}} \text { : }
$$

Now, if the constant $c$ in $r_{k}=c 2 j_{k}$ is chosen small enough (depending on ), and $x 2 B^{k} \quad B\left(x_{k} ; 100 r_{k}\right)$, one can check that

$z_{k} 2$

$X^{+}\left(X ; W_{;} ; 2^{j_{k}+1} ; 2^{j_{k}}{ }^{1+1}\right)$;

12f $1 ; 0 ; 19$

see Figure 2. In particular, one of the three scales $2^{j_{k}+1}$, I 2 f $1 ; 0$; $1 \mathrm{~g}$, is among

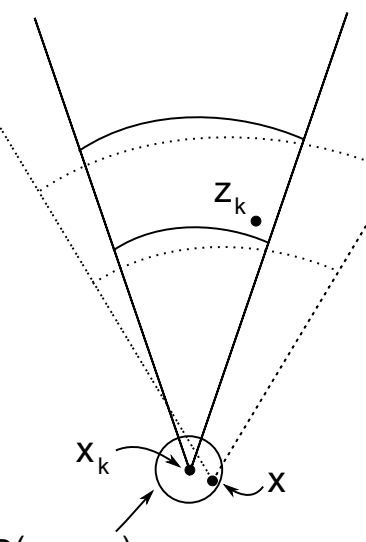

FIGURE 2. Some of the points and regions associated with $D^{k}$.

the at most $M$ scales $2{ }^{m}$ such that $X^{+}\left(x ; w ; ; 2 m^{m} ;{ }^{m}{ }^{1}\right) \backslash F^{k} G ;$. Then $D^{k}$ certainly contains all the points in the intersection $X^{+}\left(X ; w ; \quad=2 ; 2 \mathrm{~m}_{2} \mathrm{~m}^{1}\right) \backslash \mathrm{F}^{k}$, so

$$
\mathrm{X}^{+}\left(\mathrm{x} ; \mathrm{w} ; \quad 2 ; 2^{\mathrm{m}} ; 2^{\mathrm{m}^{1}}\right) \backslash \mathrm{F}^{\mathrm{k}+1}=\mathrm{X}^{+}\left(\mathrm{x} ; \mathrm{w} ; \quad 2 ; 2^{\mathrm{m}} ; 2^{\mathrm{m}^{1}}\right) \backslash\left(\mathrm{F}^{\mathrm{k}} \mathrm{nD}^{\mathrm{k}}\right)=;:
$$


Thus, there can only remain at most $M \quad 1$ scales $2 \mathrm{~m}$ such that

$$
\mathrm{X}^{+}\left(\mathrm{x} ; \mathrm{w} ; \quad=2 ; 2^{\mathrm{m}} ; 2^{\mathrm{m}}{ }^{1}\right) \backslash \mathrm{F}^{\mathrm{k}+1} G ; ; \quad \mathrm{x} 2 \mathrm{~B}^{\mathrm{k}} ;
$$

and this is exactly what is claimed at the end of (IV).

Finally, we establish the remaining claims in (III) by proving that $\mathrm{D}^{\mathrm{k}}$ is disjoint from the saved sets $S^{i}, i \quad k$. In fact, this implies that $S^{i} \backslash D^{1}=$; for all pairs $i$; $k$. Indeed, if $i \quad I<k$, we may assume by induction that $S^{i}$ is disjoint from $D^{\prime}$ (since this is precisely what we are about to prove for $I=k$ ). Further, $S^{i}$ is disjoint from $D^{\prime}$ with $\mathrm{I}<\mathrm{i}$ simply because

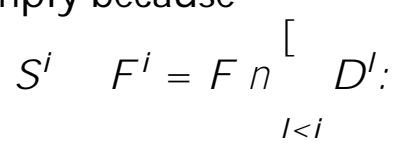

From the previous discussion, we conclude that

$$
{ }_{i k}^{i} \quad F{ }^{[} D_{k} D^{\prime}=F^{k+1} \text { : }
$$

Observe that for every $\times 2{ }^{i{ }^{k}} S_{k} S^{i}$ there are at most M 1 scales $2{ }^{j}$ such that

$$
\mathrm{X}^{+}\left(\mathrm{x} ; \mathrm{w} ;=2 ; 2^{\mathrm{j}} ; 2^{\mathrm{j}}{ }^{1}\right) \backslash \mathrm{F}^{\mathrm{k}+1} \mathrm{G} ;:
$$

This follows from (3.12) for $\times 2 S^{k} \quad B^{k}$, and from induction for $\times 2 S^{i} \quad B^{i}$ for $\mathrm{i}<\mathrm{k}$ (recalling (3.11) and noting that $F_{k+1} \quad F_{k}$ ). Hence, we infer from the inclusion (3.13) that there are also at most $M \quad 1$ scales $2{ }^{j}$ such that

$$
\mathrm{X}^{+}\left(\mathrm{x} ; \mathrm{w} ;=2 ; 2^{j} ; 2^{j}{ }^{1}\right) \backslash \prod_{\mathrm{i} k}^{\mathrm{S}} \mathrm{S}^{\mathrm{i}} \boldsymbol{\mathrm { k }}
$$

for $\times 2^{S}{ }_{i k} S^{i}$. This is what was claimed at the end of (III).

Now, we fix $i \quad k$, and establish that $D^{k}$ is disjoint from $S^{i}$. If $i=k$, this is immediate from the construction (recall that $S^{k} \quad B\left(x_{k} ; r_{k}\right)$, whereas $D^{k}$ lies inside the union of certain annuli, all at distance $r_{k}$ from $\left.x_{k}\right)$. So, we assume that $\mathrm{i}<\mathrm{k}$. There are two cases to consider. First, assume that 100 $r_{k} \quad r_{i}$ (see Figure 3$)$. In this case, we simply prove that if $x 2 B^{k} \quad B\left(x_{k} ; 100 r_{k}\right) \quad B\left(x_{k} ; r_{i}\right)$, then

$$
X^{+}(x ; w ;) \backslash B\left(x_{i} ; r_{i}\right)=; ;
$$

which is clearly a stronger statement than $D^{k} \backslash S^{i}=;$. Fix $x 2 B^{k}$, and recall that $x_{k} Z B^{i}=B\left(x_{i} ; 100 r_{i}\right) \backslash F^{i}$ for $i<k$ (because $x_{k} 2 S_{k}$, and $S_{k}$ is disjoint from $B^{i}$, as remarked below (3.10)). Because $x_{k} 2 S^{k} \quad F^{k} \quad F^{i}$, this implies that $x_{k} z B\left(x_{i} ; 100 r_{i}\right)$, and hence, by $100 r_{k} r_{i}$,

$$
x z B\left(x_{i} ; 50 r_{i}\right):
$$

Now, recall that the last coordinate of $x_{k}$ is no smaller than the last coordinate of $x_{i}$ by (IV). So, if we write $y=\left(y^{u}\right)_{u=1}^{d}$ for a general point y $2 R^{d}$, we have

$$
\begin{array}{lllll}
x^{d} & x_{k}^{d} & r_{i} & x_{i}^{d} & r_{i} \text { : }
\end{array}
$$




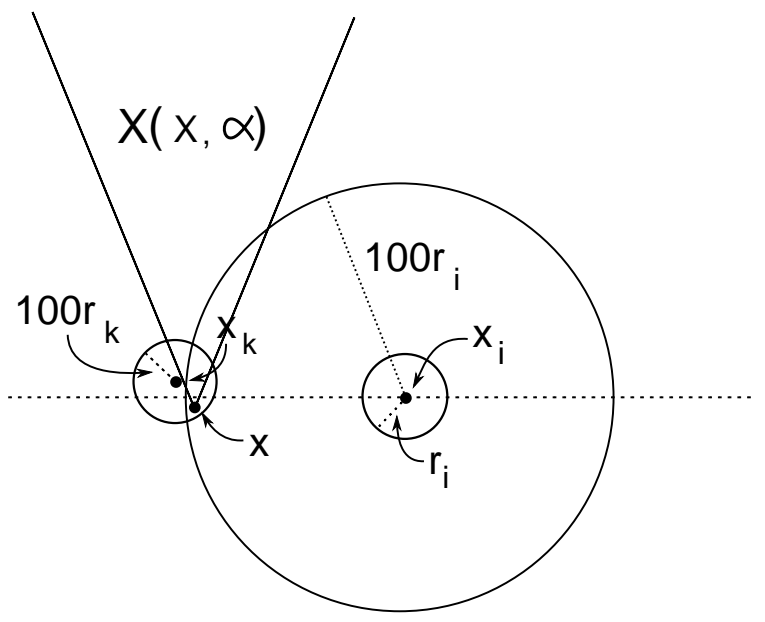

FIGURE 3. The case $100 r_{k} r_{i}$.

It is now easy to check, based on (3.15) and (3.16) that (3.14) holds, if we assume that, say, $\quad 1=10$.

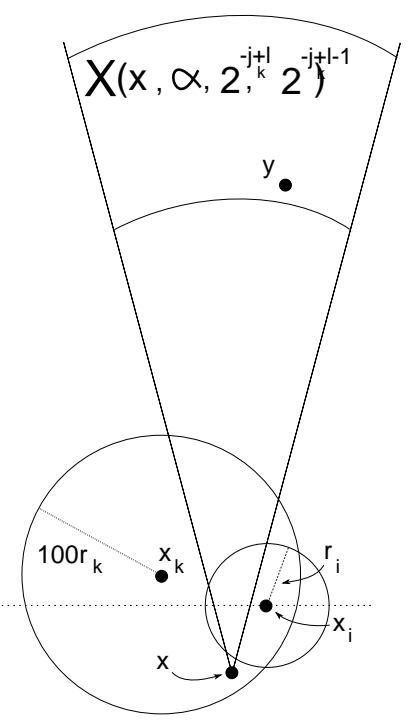

FIGURE 4. The case $100 r_{k}>r_{i}$.

Next, assume that $100 r_{k}>r_{i}$ (see Figure 4). Recall that $r_{k}=c 2 j_{k}$, and $D^{k}$ is contained in the union of the annuli $X\left(x ; w_{;} ; 2^{j_{k}+l} ; 2^{j_{k}}{ }^{1+1}\right)$, where $x 2 B^{k}$ $\mathrm{B}\left(\mathrm{X}_{\mathrm{k}} ; 100 \mathrm{r}_{\mathrm{k}}\right)$ and I $2 \mathrm{f} 1 ; 0 ; 1 \mathrm{~g}$. If $\times 2 \mathrm{~B}^{\mathrm{k}}$ is fixed, then by the same argument that gave (3.16), we now have

$$
x^{d} \quad x_{k}^{d} \quad 100 r_{k} \quad x_{i}^{d} \quad 10002^{j_{k}} \text { : }
$$


If

$$
\text { y } \left.2 \text { X (x; w; ; } 2^{j_{k}+1} ; 2^{j_{k}}{ }^{1+1}\right) \text {; }
$$

then, using (3.17) and choosing $c>0$ small enough,

$$
y^{d} \quad x^{d}+2^{j_{k}} \quad 10 \quad x_{i}^{d}+2^{j_{k}} \quad 20 \quad x_{i}^{d}+1000 c 2^{j_{k}} \quad x_{i}^{d}+10 r_{i}:
$$

In particular, $y$ cannot lie in $B\left(x_{i} ; r_{i}\right) \quad S^{i}$, and the proof is complete.

\section{A ppendix A. A measure estimate on the GRASSMANNIAN}

This section contains the proof of Lemma 2.2. Let us recall the statement:

Lemma A.1. Fix ; $>0$ and let W $2 \mathrm{G}(\mathrm{d} ; \mathrm{n})$. Assumethat z $2 \mathrm{R}^{\mathrm{d}}$ satisfies $\quad j \mathrm{j} \mathrm{j}<$ $\min (0 ;=2)$, where 0 is a small constant depending only on $d$, and j $w$ zj ojzj for a small enough $0=0(n ; \quad)>0$. Define $B_{z}=f \vee 2 G(d ; n): j \vee z j \quad g$. Then,

$$
A(z):={ }_{d ; n}\left(B_{z} \backslash B_{G(d ; n)}(W ;)\right) \& \quad \overline{j z j}^{n}:
$$

Proof. Let us begin by showing that there exists $V_{0} 2 B_{G(d ; n)}(W ; \Rightarrow)$ for which $v_{0} z=0$. Let $e_{1} ;::: ; e_{n} 2 R^{d}$ be an orthonormal basis for $W$. N otice that for every j 2 f 1 ; : : : ; ng we have that

$$
\text { jz ej j wzj ojzj: }
$$

Theplan is now to form new vectors $u_{1} ;::: ; u_{n}$ by perturbing the vectors $e_{1} ;:: ; ; e_{n}$ slightly. Let $u_{0}:=e_{0}:=z$ zzj, $z_{1}:=$; and $0:=0$. Assume that $u_{0} ;:: ; ; u_{k}$, $0 \mathrm{k}<\mathrm{n}$, have al ready been defined so that they satisfy:

(1) $\left(u_{0} ; u_{1} ;::: ; u_{k}\right)$ is an orthonormal sequence;

(2) If $Z_{i}=\operatorname{span}\left(u_{0} ; u_{1} ;::: ; u_{i}\right)$ for i 2 f $0 ;::: ; k g$ then

$$
u_{i}=\frac{z_{i}^{?}{ }_{1} e}{j z_{i}^{?} e_{1} j}
$$

and

$$
j z_{i} e_{+1} j \quad i:
$$

As will be apparent in a moment, the numbers ; will be defined via a simple recurrence relation. Observe that (A.2) also gives

$$
\text { j } z_{i} e_{+1} j \quad\left(\begin{array}{ll}
1 & 2
\end{array}\right)^{1=2} ; \quad \text { i } 2 \text { f0;:: :; kg: }
$$

Now, let

$$
u_{k+1}=\frac{z_{k}^{?} e_{k+1}}{j z_{k}^{?} e_{k+1}^{j}}
$$


and $Z_{k+1}=\operatorname{span}\left(Z_{k} ; u_{k+1}\right)$. If $k=n \quad 1$, the vectors $f u_{1} ;:: ; u_{n} g$ have now been defined, and the induction terminates. If $k<n \quad 1$, notice that

$$
\begin{aligned}
& j z_{k+1} e_{k+2} j^{2}={ }_{i=0}^{x^{+1}}\left(e_{k+2} u_{i}\right)^{2} \\
& =\left(\begin{array}{ll}
e_{k+2} & u_{0}
\end{array}\right)^{2}+{ }_{i=1}^{x+1} e_{k+2} \frac{z_{i}^{2} e_{j}}{j z_{i}^{2} e_{1} j}{ }^{2} \\
& =\left(\begin{array}{lll}
e_{k+2} & u_{0}
\end{array}\right)^{2}+{ }_{i=1}^{x+1} e_{k+2}{\frac{z_{i}{ }_{1}}{j} z_{i}^{2} e_{1}^{j}}^{2} \\
& { }_{0}^{2}+{ }_{i=1}^{x+1} \frac{i_{i}^{2}}{1} i_{i}^{2}=:{ }_{k+1}^{2}:
\end{aligned}
$$

Now, properties (1) and (2) have been verified for the vectors $u_{0} ;:: ; ; u_{k+1}$.

Define $v_{0}=\operatorname{span}\left(u_{1} ;:: ; ; u_{n}\right)$. By (1) it follows that $v_{0} z=0$. Therefore, it remains to show that $\mathrm{V}_{0} 2 \mathrm{~B}_{\mathrm{G}(\mathrm{d} ; n)}(\mathrm{W} ; \Rightarrow)$ for a small enough 0 . First, it is easy to check using the definition of $\mathrm{u}_{\mathrm{i}}$ that

$$
u_{i} \quad e_{i}=\frac{z_{i_{1}} e}{j z_{i}^{?} e_{j}^{j}}+\frac{1}{j z_{i}^{?} e j} \quad 1 \text { e: }
$$

It follows that

$$
j u_{i} \text { ej } \frac{i_{1}}{\left(1 i_{i}^{2}\right)^{1-2}}+\frac{1}{\left(\begin{array}{ll}
2 & i_{1}
\end{array}\right)^{1=2}} \quad 1=: r_{i}:
$$

Choose 0 so small that maxf $r_{i}: i=1 ;:: ; n g \quad \Rightarrow\left(4 n^{1=2}\right)$; this can clearly be done, given that the numbers i satisfy the recurrence relation above. It follows that

$$
k W \quad V_{0} k_{G(d ; n)}=k w \quad v_{0} k \quad=4 ;
$$

ending the proof of the existence of $V_{0}$.

Let $F=f V 2 G(d ; n): \quad v z=0 g$ and identify $F$ with $G(d \quad 1 ; n)$ (notice that $F$ is exactly the n-planes contained in $z^{\text {? }} \quad R^{d}{ }^{1}$ ). N ow $V_{0} 2 F$. Let $H$ bea maximal

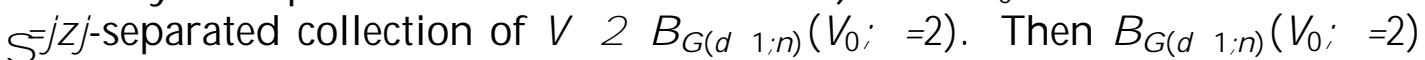

$f B_{G(d \quad 1 ; n)}(V ; 2$ jzj): $V 2$ Hgyielding that

$$
\text { 1. \#H } \overline{j z j}^{n(d}{ }^{n} \text { : }
$$

Here we used that $\left.\mathrm{d}_{1 ; n}\left(\mathrm{~B}_{\mathrm{G}(\mathrm{d}} 1 ; n\right)\left(\mathrm{V}_{0} ; \Rightarrow\right)\right) \quad 1$ and Proposition 4.1 of [5] (we also implicitly used $\neq z \mathrm{j}<0$, which, for small enough 0 , guarantees that Proposition 4.1 of [5] applies to the balls $B_{G(d \quad 1 ; n)}(V ; 2$ jzj)). N otice that

$$
\left.{ }_{\mathrm{V} 2 \mathrm{H}} \mathrm{B}_{\mathrm{G}(\mathrm{d} ; \mathrm{n})}\left(\mathrm{V}_{;}=2 \mathrm{jzj}\right)\right) \quad \mathrm{B}_{\mathrm{Z}} \backslash \mathrm{B}_{\mathrm{G}(\mathrm{d} ; \mathrm{n})}(\mathrm{W} ; \quad)
$$


by the definition of $B_{z}$, the inclusion $H \quad F$, and the inequalities $=(2 j z j) \quad=4$, and

$$
k V \quad W k_{G(d ; n)} \quad k V \quad V_{0} k_{G(d ; ; n)}+k_{0} \quad W_{k_{G}(d ; n)} \quad \frac{3}{4} ; \quad V 2 H:
$$

Also, the $G(d ; n)$-balls in the union are disjoint, so
$A(z)$
$\mathrm{X}$
$\mathrm{V} 2 \mathrm{H}$
$\left.\mathrm{d} ; \mathrm{n}\left(\mathrm{B}_{\mathrm{G}(\mathrm{d} ; \mathrm{n})}(\mathrm{V} ; \quad=2 \mathrm{j} \mathrm{zj})\right)\right) \&$
$n(d n)$
$\overline{\mathrm{jzj}}$
$\overline{\mathrm{jzj}}$
${ }^{n\left(\begin{array}{lll}d & 1 & n\end{array}\right)=\frac{n}{j z j}} \quad ;$

using the cardinality estimate for $\mathrm{H}$, and Proposition 4.1 of [5] again.

\section{REFERENCES}

[1] Jonas Azzam and Raanan Schul, Hard Sard: quantitativeimplicit function and extension theorems for Lipschitz maps, Geom. Funct. Anal. 22 (2012), no. 5, 1062-1123.

[2] Guy David and Stephen Semmes, Analysis of and on uniformly rectifiable sets, Mathematical Surveys and Monographs, vol. 38, A merican Mathematical Society, Providence, RI, 1993.

[3] __ Singular integrals and rectifiable sets in $\mathrm{R}^{\mathrm{n}}$ : Beyond Lipschitz graphs, Astérisque 193 (1991), 152.

[4] __ Quantitative rectifiability and Lipschitz mappings, Trans. A mer. Math. Soc. 337 (1993), no. 2, 855-889.

[5] Katrin Fässler and Tuomas Orponen, Constancy results for special families of projections, Math. Proc. Cambridge Philos. Soc. 154 (2013), no. 3, 549-568.

[6] Herbert Federer, Geometric MeesureTheory, Springer-Verlag, 1969.

[7] Pertti Mattila, Geometry of sets and measures in Eudidean spaces: Fractals and rectifiability, Cambridge Studies in Advanced Mathematics, vol. 44, Cambridge University Press, Cambridge, 1995.

[8] Elias M. Stein, Harmonic Analysis: Real-variableM ethods, Orthogonality, and Oscillatory Integrals, Princeton University Press, Princeton, New Jersey, 1993.

[9] Terence Tao, A quantitative version of the Besicovitch projection theorem via multiscale analysis, Proc. London Math. Soc. 98 (2009), no. 3, 559-584.

(H.M.) Department of Mathematics and Statistics, University of Helsinki, P.O.B.

68, FI-00014 HELSINKI, FINLAND

E-mail address: henri. mart i ka i nen @hel sinki . fi

(T.O.) Department of Mathematics and Statistics, University of Helsinki, P.O.B.

68, FI-00014 HELSINKI, FINLAND

E-mail address: t u o mas. or ponen@hel si nki.f i 